\title{
Philonsorbonne
}

2 | 2008

Année 2007-2008

\section{Wittgenstein et la reconnaissance d'Autrui (entre perception et concept)}

\section{Chiara PASTORINI}

\section{(2) OpenEdition}

1 Journals

Édition électronique

URL : https://journals.openedition.org/philonsorbonne/180

DOI : 10.4000/philonsorbonne. 180

ISSN : 2270-7336

Éditeur

Publications de la Sorbonne

\section{Édition imprimée}

Date de publication : 15 avril 2008

Pagination : 111-129

ISBN : 978-2-85944-602-4

ISSN : 1255-183X

\section{Référence électronique}

Chiara PASTORINI, «Wittgenstein et la reconnaissance d'Autrui (entre perception et concept) », Philonsorbonne [En ligne], 2 | 2008, mis en ligne le 28 janvier 2013, consulté le 08 juin 2021. URL http://journals.openedition.org/philonsorbonne/180; DOI : https://doi.org/10.4000/philonsorbonne. 180

\section{(C) Tous droits réservés}




\title{
Wittgenstein et la reconnaissance d'Autrui (entre perception et concept)
}

\author{
Chiara Pastorini
}

Le but essentiel de cet article est d'analyser la notion de «relation intersubjective » chez Wittgenstein. En particulier, nous nous proposons de découvrir si le philosophe aborde la relation Moi-Autrui en termes épistémiques, c'est-à-dire dans une dimension conceptuelle, ou s'il déplace la question sur une dimension différente.

À ce propos, nous montrerons que, loin d'être considérée au niveau épistémique de la connaissance conceptuelle, la relation Moi-Autrui est déplacée par Wittgenstein au niveau pratique anthropologique de la reconnaissance (niveau des jeux de langage). Une reconnaissance immédiate (c'est-à-dire sans la médiation du raisonnement) remplace la perspective traditionnelle (cartésienne) d'une attitude conceptuelle (de type sceptique, basée sur les critères du vrai et du faux) et d'interprétation de l'autre.

Les notions qui définissent l'espace de l'intersubjectivité selon la perspective de Wittgenstein sont les suivantes: «reconnaissance » (Anerkennung), «accord 》 (Übereinstimmung) «phénomène originaire » (Urphänomen), « apprentissage » (Abrichtung), « règle» (Regel), «voir comme (sehen als). Wittgenstein ne parle pas explicitement de «corps » (Körper) comme d'un fondement de la relation Moi-Autrui, mais les notions de «phénomène originaire », de «forme de vie », de «primitif», d' «instinctif», d' "activité synesthésique » et même du «voir comme» renvoient, selon notre interprétation, à une matrice corporelle (et non pas conceptuelle) du rapport intersubjectif.

Les œuvres wittgensteiniennes suivantes sont des sources particulièrement riches pour cette analyse: Bemerkungen über die Philosophie der Psychologie, Philosophische Untersuchungen, Über Gewissheit, Zettel. 


\section{Connaissance ou reconnaissance d'autrui ?}

Dans un cadre théorique traditionnel la notion de «relation intersubjective » se pose comme un problème de connaissance de l'esprit des autres.

Et c'est bien la connaissance des autres qui oriente et définit plus que toute autre question le cours de la philosophie moderne. C'est à partir de la naissance de la subjectivité moderne, c'est-à-dire à partir de la distinction cartésienne entre une substance spirituelle (res cogitans) et une substance corporelle (res extensa), que le problème de l'être est reformulé avec l'opposition entre la figure d'un sujet de connaissance d'un côté et un objet connu de l'autre côté. Le sujet devient dans le cartésianisme la seule évidence absolue, soit d'un point de vue métaphysique, soit d'un point de vue méthodologique ${ }^{1}$. L'accès à l'autre, donc, n'est possible qu'à partir d'un modèle d'analyse centré sur la recherche d'une technique pour la vérification de l'objet (l'autre). Dans cette perspective l'esprit des autres assume le rôle d'une entité quelconque, pouvant être soumise à une technique de vérification qui rend possible la description, le calcul, la prévision de l'objet même (l'esprit - le pas vers le contrôle sur Autrui peut ne pas être grand).

Dans ce type d'approche dualiste de l'autre, selon une tradition qui va de René Descartes à John Locke, et qui continue jusqu'à une certaine philosophie contemporaine de l'esprit, le but devient d'établir avec certitude les conditions nécessaires et suffisantes à l'identification de l'état mental d'Autrui, non pas seulement pour ce qui concerne son existence effective, mais aussi pour ce qui est de ses contenus mêmes, normalement renfermés en chacun, invisibles et cachés aux autres. Cette attitude, qui remonte à Descartes, traduit la relation Moi-Autrui en termes épistémiques d'un rapport de connaissance. Autrement dit, la relation intersubjective s'inscrit dans une dimension conceptuelle où la représentation de l'objet intervient comme élément médiateur entre le sujet de la connaissance (le Moi) et l'objet même (Autrui).

Pour ce qui est de la philosophie contemporaine de l'esprit, nous pouvons considérer trois grands courants : le mentalisme, le béhaviourisme et le solipsisme. Bien qu'elles aient le même présupposé et considèrent la relation Moi-Autrui dans l'espace conceptuel du raisonnement et de l'interprétation, ces trois perspectives théoriques adoptent trois modalités différentes d'accès à l'autre : en termes grossiers, il est possible affirmer que le mentalisme réduit l'autre à de purs états mentaux ${ }^{2}$, le béhaviourisme à de purs comportements et le solipsisme, bien qu'il considère l'autre comme un

1. Cf. R. Descartes, Discours de la méthode (1637), Paris, Hatier, 1999.

2. Dans cette acception le mentalisme est entendu comme une famille de théories postulant un esprit, généralement substantiel, comme sujet d'activités mentales ou comme lieu où celles-ci se déroulent (cf. C. Chauviré, Voir le visible. La seconde philosophie de Wittgenstein, Paris, PUF, 2003, Glossaire, p. 5). 
objet de connaissance, nie toute possibilité d'accéder à lui. Les approches de type cartésien (qu'elles soient mentaliste, behaviouriste ou solipsiste), en tant que modalité conceptuelle d'accès à l'autre, représentent la cible de la philosophie wittgensteinienne ${ }^{3}$.

En faisant référence à l'interprétation de Stanley Cavell dans The Claim of Reason ${ }^{4}$, il est possible d'affirmer que la position traditionnelle (cartésienne) du problème de l'esprit des autres en termes épistémiques contient le germe d'une attitude de type sceptique qui se traduit par une tendance à s'exposer aux tentations théoriques. Cette attitude, considérée comme une exigence humaine inextinguible, est reconnue par Wittgenstein comme une volonté de sublimation linguistique :

«Diese seltsame Auffassung rührt von einer Tendenz her, die Logik unserer Sprache zu sublimieren $»^{5}$.

Cette volonté de sublimation se retrouve dans toute position qui considère les critères d'identification humaine non seulement comme la limite de notre connaissance, mais aussi comme notre limitation (d'être humain). D'où la nécessité de chercher des critères absolus, surhumains, susceptibles d'exorciser et de dépasser les doutes et l'incertitude qui caractérisent notre condition d'être humain, recherche ayant pour conséquence l'éloignement du langage ordinaire et l'aliénation des pratiques linguistiques de la communauté. Autrement dit, dans l'interprétation de Cavell, le doute sceptique, constitutif de l'être humain en tant qu'incertitude quant à la possibilité d'une connaissance réelle de l'esprit de l'autre, nous mène, à travers une opération de sublimation, à la recherche de supercritères qui, en dépassant l'ordinaire de notre vie, se surélèvent à une dimension autre. Cette dimension garantit à son tour nos relations mondaines intersubjectives.

Contre les super-critères et les super-concepts (Über-Ordnung, ÜberBegriffen PU, I, § 97) d'une vision intellectualisée du langage, immobilisée en formes rigides et paradigmatiques, Wittgenstein en appelle à un langage ordinaire, gouverné par les règles (Regeln) quotidiennement utilisées dans les pratiques intersubjectives humaines. Comme J. Bouveresse le souligne dans Le mythe de l'intériorité, la philosophie du langage de Wittgenstein s'intéresse «au phénomène spatial et temporel du langage, et non pas à

3. Cf. N. Malcolm, Problems of Mind. Descartes to Wittgenstein, New York, Harper \& Row, 1971.

4. Cf. S. Cavell, The Claim of Reason. Wittgenstein, Skepticism, Morality, and Tragedy, New York, Oxford University Press, 1979.

5. L. Wittgenstein, Philosophische Untersuchungen. Philosophical Investigations [I, 19361945 ; II, 1947-1949], éd. par G. E. M. Anscombe et R. Rhees, trad. G. E. Anscombe, Oxford, Basil Blackwell, 1953, 20013 , I, § 38 ; trad. fr. : «Cette étrange conception provient d'une tendance à sublimer - pourrait-on dire - la logique de notre langage », L. Wittgenstein, Recherches philosophiques, traduit de l'allemand par F. Dastur, M. Élie, J.-L. Gautero, D. Janicaud, É. Rigal, Gallimard, Paris, 2004, I, § 38. 
une réalité translinguistique inobservable et sublime qui serait à l'œuvre derrière le langage $»$.

Wittgenstein écrit dans ses Philosophische Untersuchungen :

«Wenn ich über Sprache (Wort, Satz, etc) rede, muß ich die Sprache des Alltags reden $»^{7}$.

Le raidissement dans une forme paradigmatique augmente les distances entre les individus et une connexion avec le monde (et avec les individus qui y habitent) caractérisée par la tentative de dépasser (et donc de méconnaître) les formes de vie ordinaire où cette connexion est déjà établie depuis toujours devient impossible. En d'autres termes, c'est seulement à l'intérieur des règles pratiques et partagées des jeux de langage (Sprachspiel ou language-game) que l'on délimite, sans en établir forcément la limite (en tant qu'humain), la possibilité de nos relations intersubjectives. La règle rappelle les locuteurs partageant les même pratiques d'usage à une intersubjectivité ordinaire qui naît d'un accord fondamental (Übereinstimmung, PU, I, § 224) entre les êtres humains dans le langage ${ }^{8}$. Et c'est bien à travers la notion de «règle » que Wittgenstein déconstruit le doute sceptique dans son fondement métaphysique, en le réduisant à un jeu entre les autres :

«Das Spiel des Zweifelns selbst setzt schon die Gewißheit voraus » ${ }^{9}$.

Le doute est ainsi pourvu de la connotation d'absoluité et de l'aspiration à la totalité qui sont présentes au contraire dans l'attitude sceptique dominant la philosophie moderne. Ce déplacement par rapport au niveau de connaissance permet de mettre en évidence les termes d'une question traditionnellement mal posée :

«'Wissen' und 'Sicherheit' gehören zu verschiedenen Kategorien » ${ }^{10}$.

Savoir et certitude appartiennent à deux catégories différentes.

6. Cf. J. Bouveresse, Le mythe de l'intériorité. Expérience, signification et langage privé chez. Wittgenstein, Paris, Minuit, 1976, $1987^{2}$, p. 220.

7. L. Wittgenstein, Philosophische Untersuchungen, op. cit., I, § 120 ; tr. fr. : « Si je parle du langage (du mot, de la proposition, etc.), il me faut parler le langage de tous les jours », op. cit., § 120.

8. Wittgenstein écrit : «Das Wort "Übereinstimmung" und das Wort "Regel" sind miteinander verwandt, sie sind Vettern », PU, I, § 224 ; tr. fr. : «Les mots "accord" et "règle" sont apparentés, ils sont cousins », op. cit., I, § 224.

9. Id., Über Gewissheit. On Certainty [1950-1951], éd. par G. E. M. Anscombe et G. H. Von Wright, tr. par G. E. Anscombe et Denis Paul, New York, Harper \& Row, 1972 $\S 115$; tr. fr. : «Le jeu du doute lui-même présuppose la certitude », L. Wittgenstein, De la certitude, tr. de l'allemand par J. Fauve, Gallimard, Paris, 1965, 1976², § 115.

10. Ibid., op. cit., § 308 ; tr. fr. : «"savoir" et "certitude" appartiennent à deux catégories différentes », op. cit., $§ 308$. 


\section{Et encore :}

«Das Wissen gründet sich am Schluß auf der Anerkennung » ${ }^{11}$.

En d'autres termes, notre relation avec le monde et avec les autres esprits n'est pas réductible à un certain degré de connaissance, de savoir et de certitude cognitive, comme le croit le sceptique, mais se manifeste plutôt comme une reconnaissance qui se structure par les règles pratiques de nos formes de vie humaine et qui considère tout ordre externe aux telles pratiques comme une réification indue. Ainsi, les rapports intersubjectifs se basent sur une capacité et une volonté réciproque d'expression ou de réponse à l'autre, et la pratique humaine de la reconnaissance (Anerkennung) prend en charge, par rapport à toute justification de type épistémique, un rôle fondateur ${ }^{12}$.

\section{La reconnaissance comme phénomène originaire}

Dans le but de montrer la nature originaire et non médiatisée par des éléments conceptuels de la reconnaissance intersubjective, il paraît pertinent de s'interroger sur la conception wittgensteinienne du rapport Moi-Autrui, en prenant en considération cette remarque du philosophe :

" "Ich glaube, daß er leidet". - Glaube ich auch, daß er kein Automat ist ?

Nur mit Widerstreben könnte ich das Wort in diesen beiden Zusammenhängen aussprechen $»^{13}$.

En se rapportant au contexte de la douleur humaine, situation sur laquelle Wittgenstein réfléchit en de nombreux endroits, le philosophe montre comment, dans l'approche d'une personne qui souffre, la croyance et tout ce qui appartient à la sphère de l'opinion, du calcul et, en un mot, de la rationalité conceptuelle, sont mis de côté (tout cela « hat, so ohne weiteres,

11. Ibid, op. cit., § 378. En anglais, « Knowledge is in the end based on acnowledgement »; tr. fr. : «Le savoir en fin de compte est basé sur la reconnaissance », op. cit., § 378.

12. À propos de la notion de «reconnaissance » comme fondement éthique, voir D. Sparti, L'importanza di essere umani. Etica del riconoscimento, Milan, Feltrinelli, 2003. Sparti utilise le terme response-ability pour définir cette éthique de la reconnaissance. Comme le même auteur le remarque (Ibid., p. 171), le mot "responsabilité » a pour racine latine spondere et, plus originairement la racine grecque spondein. Les deux déclinaisons font référence à un domaine sémantique plus ample que le sens juridique actuel (responsabilité conçue comme le fait de rendre raison de ses actes en tant que sujet moral), en renvoyant plutôt à la capacité la plus générale de répondre à un appel.

13. L. Wittgenstein, Philosophische Untersuchungen, II, ibid., p. 152 ; tr. fr. : " "Je crois qu'il souffre." - Est-ce que je crois aussi qu'il n'est pas un automate ? Je ne pourrais employer qu'à contrecœur le mot "croire" dans ces deux contextes », op. cit., II, ibid., p. 253. 
noch gar keinen Sinn », PU, II, iv, p. $\left.152^{14}\right)$. La souffrance, en tant que particularité humaine ( Nur von dem, was sich benimmt wie ein Mensch, kann man sagen, daß es Schmerzen hat. Denn man muß es von einem Körper sagen, oder, wenn du willst, von einer Seele, die ein Körper hat », PU, I, $\S 283^{15}$ ), représente une évidence immédiate pour celui qui y assiste. L'expérience de la douleur de l'autre est, en ce sens, plus originaire, plus primitive que tout raisonnement et que toute conceptualisation.

On ne considère pas les autres comme des êtres humains à la suite de la réflexion ${ }^{16}$ (Überlegung) qu'ils sont doués d'un corps (ou d'une âme) et qu'ils ne sont donc pas des machines ou des automates, mais on formule des opinions, des jugements et des conjectures seulement d'après une coappartenance originaire dans laquelle le rapport Moi-Autrui est quelque chose de primitif : « Ich könnte von einem Menschen ja immer sagen, er sei ein Automat (das könnte ich so in der Schule beim Physiologieunterricht lernen) und es würde doch meine Einstellung zum Andern nicht beeinflussen. Ich kann es ja eben auch von mir selber sagen ${ }^{17}$. On ne croit pas que l'autre souffre ou qu'il est heureux à la suite d'une connaissance, donc, mais à travers une reconnaissance immédiate, c'est-à-dire, sans la médiation conceptuelle d'une pensée ${ }^{18}$.

Comme le paragraphe 1 le suggérait déjà le problème de l'intersubjectivité, par conséquent, ne peut pas être abordé au niveau épistémique de la connaissance conceptuelle mais, selon la perspective

14. Tr. fr. : «sans plus de précisions, n'a pas encore le moindre sens », op. cit., II, ibid., p. 253.

15. Tr. fr. : «Ce n'est que d'une chose qui se comporte comme un être humain qu'on peut dire qu'elle a mal. Car c'est d'un corps, ou, si tu préfères, d'une âme qu' $a$ un corps, qu'il faut le dire », op. cit., I, § 283.

16. Cf. L. Wittgenstein, Bemerkungen über die Philosophie der Psychologie. Remarks on the Philosophy of Psychology [1946-1949; 1947-1948], éd. par G. E. M. Anscombe et G. H. Von Wright, rad. par G. E. Anscombe, 2 vol., Oxford, Basil Blackwell, 1980, 1988², II, § 632: «Ich will eigentlich sagen, dass die gedanklichen Skrupel im Instinkt anfangen (ihre Wurzeln haben). Oder auch so: Das Sprachspiel hat seinen Ursprung nicht in der Überlegung », tr. fr. : «Ce que je cherche à dire, c'est que les scrupules de pensée ont leur commencement (leurs racines) dans l'instinct. Sous une autre forme : Le jeu de langage n'a pas son origine dans la réflexion », L. Wittgenstein, Remarques sur la Philosophie de la Psychologie, tr. de l'allemand par G. Granel, T.E.R., Mauvezin, 1994, II, § 632.

17. L. Wittgenstein, Lezte Schriften über die Philosophie der Psychologie. Last writings on the Philosophy of Psychology. Vol. 1 Vorstudien zum zweiten Teil der "Philosophische Untersuchungen". Preliminary Studies for Part II of the "Philosophical Investigation" [19481949]; vol. 2 Das Innere und das Äussere. The Inner and Outer [1948-1951], éd. par G. H. von Wright et Hikky Nyma, trad. par C. J. Luckhardt et M. A. E. Aue, Chicago, The University of Chicago Press, 1982, 1990², II, p. 138, tr. fr. : «Je pourrais fort bien dire d'un homme qu'il est un automate (je pourrais apprendre à le dire à l'école, dans un cours de physiologie) sans que cela influe sur mon attitude envers autrui. Je pourrais même le dire de moi-même », L. Wittgenstein, L'intérieur et l'extérieur. Derniers écrits sur la philosophie de la psychologie, II, tr. de l'allemand par G. Granel, T.E.R., Mauvezin, 2000, p. 55.

18. Cf. L. Perissionotto, «Wittgenstein e il problema degli altri », “Aut aut”, 51, 2001, p. 16-25. 
wittgensteinienne, au niveau pratique de la reconnaissance. Dans le rapport avec l'autre toutes les possibilités de rapprochement cognitif, de falsification ou de doute sont exclues :

«Versuch einmal - in einem wirklichen Fall - die Angst, die Schmerzen des Andern zu bezweifeln !» ${ }^{19}$.

La non pertinence d'une attitude sceptique qui puisse menacer le rapport avec l'autre se renforce donc encore une fois. En outre, des réflexions comme : "Sicher sein, daß der Andre Schmerzen hat, zweifeln, ob er sie hat, u.s.f., sind soviel natürliche instinktive Arten des Verhältnisses zu den andern Menschen ${ }^{20}$, ne démentent pas tout ce qui a été affirmé sur l'interprétation erronée de l'intersubjectivité comme dimension épistémique, mais confirment plutôt le caractère instinctif du rapport Moi-Autrui. Notre langage est une extension de la relation intersubjective, une extension d'un comportement primitif alors que les jeux de langages ne sont qu'instinct (cf. Z, § 545). Le doute et la certitude, dans cette perspective, se rapportent pour Wittgenstein au jeu linguistique du vrai et du faux, un jeu entre les autres. L'attitude (Einstellung), selon Wittgenstein, vient avant l'opinion (Meinung). On ne s'adresse pas à l'autre, ou on n'essaie pas de soulager sa souffrance, parce qu'on sait, on croit ou on suppose qu'il est un être humain, mais, au contraire, en se tournant vers lui, on essaie de l'aider, de sourire, on demande à notre tour du réconfort etc., avant toute conjecture : "Ich habe nicht die Meinung, daß er reine Seele hat » (PU, II, ibid., p. $\left.152^{21}\right)$. C'est l'attitude que j'ai à l'égard de l'être humain qui précède et oriente tous mes futurs jugements, évaluations et croyances ${ }^{22}$.

19. L. Wittgenstein, Philosophische Untersuchungen, I, § 303, tr. fr. : «Essaie seulement dans un cas effectif - de mettre en doute l'angoisse ou la douleur d'autrui », op. cit., I, § 303.

20. Id., Zettel [1929-1948, 1945-1948], éd. par G. E. M. Anscombe et G. H. Von Wright, trad. par G. E. M. Anscombe, Berkley, University of California Press, $1970,1975^{2}$, $\S 545$; tr. fr. : «Être sûr qu'autrui souffre, douter qu'il souffre, et ainsi de suite, ce sont là autant de types naturels et instinctifs de notre relation à autrui », L. Wittgenstein, Fiches, tr. de l'allemand par J. Fauve, Gallimard, Paris 1970, § 545.

21. Tr. fr. : «Je ne suis pas d'avis qu'il a une âme », cit., II, ibid., p. 253.

22. La distance entre la sphère du prélangagier et celle du raisonnement interprétatif et rationnel est aussi mise en évidence par Wittgenstein à travers un autre passage de Philosophische Grammatik : "Wenn man mich fragt "wie viel Uhr ist es ?", so geht in mir kine Arbeit des Deutens vor ; sondern ich reagiere einfach auf das, was ich sehe und höre. Es zückt Einer das Messer auf mich, dann sage ich nicht: "ich deute das als eine Drohung" » (L. Wittgenstein, Philosophische Grammatik. Philosofical Grammar [1929-1934; 19341935], in Werkausgabe Band 4, Frankfurt am Main, Suhrkamp, 1984, I, § 9) ; tr. fr. : « Quand on me demande "quelle heure est-il ?", il ne se déroule en moi aucun processus d'interprétation, je réagis simplement à ce que je vois et entends. Si quelqu'un brandit un couteau contre moi je ne dis pas: "j'interprète ce signe comme une menace" » (L. Wittgenstein, Grammaire philosophique, traduit de l'allemand par M.-A. Lescourret, Gallimard, Paris, 1980, I, § 9). Cela ne signifie pas exclure a priori des circonstances ou des contextes, réels ou imaginaires, dans lesquels il serait sensé d'affirmer : «Je l'interprète comme une menace », mais, dans ces cas là, il s'agirait de situations où dégainer un couteau serait entendu comme une plaisanterie ou un acte d'arrogance, c'est-à-dire, comme une action 


\section{Primitivité et concept}

Mais qu'entend Wittgenstein quand il parle de phénomène originaire ? En quel sens la reconnaissance peut-elle être entendue comme telle?

Pour répondre à ces questions il faut se référer à ce que le philosophe définit comme "pré-linguistique », adjectif utilisé par le même Wittgenstein pour qualifier le comportement sur lequel se base le jeu de langage, modèle pratique vital des relations intersubjectives humaines. Wittgenstein affirme dans le Bemerkungen über die Philosophie der Psychologie:

«Was aber will hier da Wort "primitiv" sagen? Doch wohl, daß die Verhaltungsweise vorsprachlich ist : dass ein Sprachspiel auf ihr beruht, dass sie das Prototyp einer Denkweise ist und nicht das Ergebnis des Denkens $\gg{ }^{23}$.

Dans cette citation il est possible de souligner, en premier lieu, la connexion entre les notions de "prélangagier» (vorsprachlich) et de «primitif» (primitiv). Le domaine sémantique de ce qu'on entend par « prélangagier» (ou pré-linguistique) est en effet étendu par Wittgenstein à des autres concepts, comme «primitif», justement, « réaction primitive » (primitive Reaktion, PU, p. 185), «phénomène originaire » (Urphänomen, PU, I, § 654), « instinct » et comportement ('Instink' et Benehmen, Z, § 545), «action » (Tat, UG, § 402) $)^{24}$, «vie » (Leben, UG § 559), «forme de vie » (Lebensform ; "Das Hinzunehmende, Gegebene- könnte man sagen- seien Lebensformen », PU, II, xi, p. $192^{25}$ ). Le pré-linguistique est donc tout ce qui est donné et qui, en même temps, constitue notre jeu, le matériel amorphe dans lequel nous sommes plongés et sur lequel le langage se structure :

«So könnte also auch aus etwas ganz Amorphen ein Organismus, sozusagen ursachelos, werden; und es ist kein Grund, warum sich dies nicht mit unserem Gedanken, also mit unserem Reden oder Schreiben etc. wirklich so verhalten sollte $»^{26}$.

dépouillée de son pouvoir originaire de produire un état de peur, ou bien, de provoquer une réaction immédiate capable d'anéantir toute faculté de raisonnement.

23. L. Wittgenstein, Bemerkungen über die Philosophie der Psychologie. Remarks on the Philosophy of Psychology, cit., I, § 916 ; tr. fr. : «Mais que veut dire ici le mot "primitif"? Bel et bien ceci : que le mode de comportement en question est prélangagier, c'est-à-dire qu'un jeu de langage repose sur lui, qu'il est le prototype d'une façon de penser et non le résultat de la pensée », op. cit., I, § 916 (Cf. aussi id., Zettel, op. cit., § 541).

24. Dans ce paragraphe, en réalité, le terme «action» est emprunté au vers du Faust de Johann Wolfgang von Goethe qui écrit : «Im Anfang war die Tat », (J. W. Goethe, Faust I, 1808, vers 1237).

25. Tr. fr. : «Ce qui doit être accepté, le donné - pourrait-on dire -, ce sont des formes de vie », op. cit., II, xi, p. 316.

26. L. Wittgenstein, Bemerkungen über die Philosophie der Psychologie, cit., I, § 903 ; tr. fr. : «Ainsi pourrait-il se faire qu'un organisme sorte de quelque chose de totalement informe, pour ainsi dire sans cause ; et il n'y a pas de raison que cet organisme doive avoir la relation qu'on lui suppose avec notre pensée, en l'occurrence avec notre parole ou notre écriture », op. cit., I, § 903 (Cf. Z, § 608). 
En second lieu, on peut constater que le pré-linguistique est un prototype (Prototyp) et non pas le résultat (Ergebnis) d'un raisonnement. Wittgenstein insiste sur le fait qu'il est fondamental comme point de départ, comme donné, et non pas comme ce à quoi on doit arriver, par exemple, à travers la logique conceptuelle de la pensée ${ }^{27}$.

En troisième lieu, il est important de mettre en évidence la relation même que le pré-linguistique a avec notre faculté de raisonnement. Ce que Wittgenstein soutient ici est que le primitif, l'action et l'instinct représentent le fondement de la rationalité humaine, sur lequel la pensée et le concept se structurent. À l'appui de cette thèse, il paraît opportun de rappeler cette citation issue des Bemerkungen über die Philosophie der Psychologie :

«Ich will eigentlich sagen, daß die gedanklichen Skrupel im Instinkt anfangen (ihre Wurzeln haben). Oder auch so: Das Sprachspiel hat seinen Ursprung nicht in der Überlegung (réflexion). Die Überlegung ist ein Teil des Sprachspiels $»^{28}$.

\section{Et encore :}

«Der Instinkt ist das Erste, das Raisonnement das Zweite. Gründe gibt es erst in einem Sprachspiel $»^{29}$.

Le pré-linguistique ou l'instinctif, de ce point de vue, devient alors synonyme d' «immédiat», au sens de non médiatisé par le raisonnement conceptuel, le calcul et les procès inductifs ou analogiques (« Die Sprache ist

27. À propos de la relation entre la dimension perceptive (pré-langagière) et la dimension épistémique (conceptuelle-langagière), Jocelyn Benoist définit l' «expérience primordiale de la perception » comme "un contact qui n'est pas un savoir, mais donne lieu à un savoir» (J. Benoist, Les limites de l'intentionnalité. Recherches phénoménologiques et analytiques, Paris, Vrin, 2005, p. 282). Toutefois, il faut rappeler qu'ici la question de la perception comme expérience primordiale est abordée dans le contexte plus ample de l'idéalisme transcendantal, qui dans l'espace perceptif même trouve sa limite.

28. L. Wittgenstein, Bemerkungen über die Philosophie der Psychologie, op. cit, II, § 632 ; tr. fr. : «Ce que je cherche à dire, c'est que les scrupules de pensée ont leur commencement (leurs racines) dans l'instinct. Sous une autre forme: Le jeu de langage n'a pas son origine dans la réflexion. La réflexion est une partie du jeu de langage », op. cit., II, § 632 (Cf. aussi $\mathrm{Z}, \S 389)$.

29. Ibid., II, § 689 ; tr. fr. : «L'instinct vient en premier, le raisonnement en second. Il n’y a de raisons que dans un jeu de langage », cit., II, § 689. À propos de cette dernière assertion, il ne faut pas interpréter Erste et Zweite, comme des critères différentiels dans une perspective ontologique ou temporelle. L'espace dans lequel la pensée wittgensteinienne se déplace est unique et constitué par la surface du langage dont les limites sont définies par ce qui est logiquement donné. Déjà dans le Tractatus Wittgenstein écrivait: «Die Tatsachen im logischen Raum sind die Welt » (TLP [1918-1921], in Werkausgabe Band 1, sous la direction de G. H. Von Wright, et G. E. M. Anscombe, Frankfurt am Main, Suhrkamp, 1964, 1989², prop. 1.13 ; tr. fr. : «Les faits dans l'espace logique sont le monde», Wittgenstein, Tractatus logico-philosophicus, tr. de l'allemand par G.-G. Granger, Gallimard, Paris, 1993, prop. 1.13). 
nicht aus einem Raisonnement hervorgegangen », UG, $\S 475^{30}$ ). En ce sens, le pré-linguistique ne peut pas être conceptualise ${ }^{31}$.

Jusqu'à maintenant nous avons parlé de «pré-linguistique » en l'identifiant à la composante primitive-instinctuelle et non médiatisée par des raisonnements dans laquelle il est possible de reconnaitre le fondement des jeux, modèles de la pratique humaine intersubjective. Après s'être rapporté au domaine du "pré-linguistique », pour la fertilité conceptuelle intrinsèque des situations limites, il peut être intéressant aussi de s'interroger sur la notion symétrique de «post-linguistique ». Wittgenstein n'utilise pas explicitement cette expression dans ses écrits, mais il est possible de trouver, en d'autres termes, des allusions claires à ce qui peut être considéré comme la réalisation extrême d'une praxis linguistique entièrement détendue ${ }^{32}$. Le résultat extrême d'une pensée rigoureuse et logiquement structurée est, même ici, une réaction primitive, au sens de non médiatisée par le raisonnement conceptuel : c'est l'expérience synesthésique vécue que l'on peut appeler expérience du «sentir comme $»^{33}$.

Cette sensibilité, qui du «voir comme» s'étend aux autres domaines sensoriels, quoique source d'une expérience immédiate est possible, dans la perspective de Wittgenstein, seulement par un fondement conceptue ${ }^{34}$. En se

30. Tr. fr. : «Le langage n'est pas issu d'un raisonnement », op. cit., § 475.

31. Cette affirmation peut aussi être considérée comme valide du point de vue de toutes les perspectives théoriques qui circonscriront le conceptuel au domaine du langage (parmi lesquelles, la théorie fregéenne du concept, conçu comme fonction dont la valeur est une valeur de vérité). Selon Frege, l'analyse fonctionnelle (et donc conceptuelle) s'étend seulement aux expressions de type linguistique. Cf. G. Frege, Funktion und Begriff (1891), H. Pohle, Iéna, 1891, tr. fr. Fonction et concept par C. Imbert, in Écrits logiques et philosophiques, Seuil, Paris, 1971, p. 80-101.

32. Cf. M. Mazzeo, P. Virno, Il fisiologico come simbolo del logico. Wittgenstein fisionomo, in Sensibilità e linguaggio. Un seminario su Wittgenstein, Macerata, Quodlibet, 2002, p. 119-155.

33. Silvana Borutti, à propos du «remarquer des aspects » d'une figure parle d' « un evento che non riguarda un solo senso », mais qui « ha piuttosto i caratteri sinestesici della capacità di "sentire la forma" in generale» (S. Borutti, Percezione e immagine. Un'estetica per le scienze umane, in "Aut aut", 313-314, p. 192).

34. À ce propos, il serait intéressant de comparer la perspective de Wittgenstein avec celle de Fred Dretske qui distingue un voir «propositionnel» (ou «épistémique») et un voir « simple », le premier traduisible dans la proposition « $\mathrm{X}$ voit que $\mathrm{X}$ est $\mathrm{Y}$ » et le deuxième en « $\mathrm{S}$ voit $\mathrm{X} » .-\mathrm{Si}$ le voir épistémique est censé être relatif aux croyances (à leur tour sujettes à l'expérience passée, à certaines habitudes d'associations etc.) (« Nothing can be seen in these ways without acquiring some true belief about what is seen », cf. F. Dretske, Seeing and Knowing, Chicago, The University of Chicago Press, 1969, p. 76), le voir simple (voir «non épistémique ») est au contraire complètement indépendant d'eux. Le philosophe décrit en fait la vision non épistémique comme dépourvue de contenu doxastique, c'est à dire de contenu de croyance, en lui donnant plutôt le caractère d'une capacité visuelle primitive («primitive visual ability», cf. F. Dretske, Seeing and Knowing, op. cit., p. 4). Selon Dretske, le voir «non épistémique » est une activité commune à une grande partie des êtres vivants et libre des influences de l'éducation, de l'expérience passée, de la sophistication linguistique et de l'astuce conceptuelle. 
référant au « voir comme » (sehen als), Wittgenstein écrit dans la deuxième partie des Philosophische Untersuchungen:

«Nur von dem würde man sagen, er sähe es jetzt so, jetzt so, der imstande ist, mit Geläufigkeit gewisse Anwendungen von der Figur zu machen.

Das Substrat dieses Erlebnisses ist das Beherrschen einer Technik ${ }^{35}$.

Seul celui qui est maître d'une technique, c'est-à-dire qui, à travers un procès d'apprentissage, devient expert d'une grammaire linguistique déterminée est donc en mesure de voir une image (un triangle, par exemple) en saisissant l'aspect (Aspekt), tantôt d'une manière, tantôt d'une autre, par exemple « jetzt das als Spitze, das als Grundlinie sehen- jetzt das als Spitze und das als Grundlinie » (PU, II, XI, p. $178^{36}$ ). Voir l'aspect est alors une catastrophe perceptive, un changement de forme (Gestalt-switch) soudain que Wittgenstein décrit en employant les verbes aufleuchten, erscheinen, entstehen: briller (au sens de venir tout dans coup à l'esprit), paraître, surgir $^{37}$. En passant du pré-linguistique à l'autre extrême du jeu dans lequel on remarque des aspects (espace du sehen als), on s'aperçoit qu'on se retrouve immergés de nouveau dans le matériel amorphe des réactions immédiates et des sensations dans lesquelles il n'y a pas de place pour la pensée ni pour la conjecture :

«Deuten ist ein Denken, ein Handeln ; Sehen ein Zustand ${ }^{38}$.

Toutefois, voir un triangle soit comme un trou triangulaire soit comme un mont soit comme une flèche c'est plus que la simple impression visuelle. Le langage, à travers le raisonnement, remplit la distance entre l'instinct et cet état (Zustand) que nous avons défini comme «post-linguistique » (en tant qu'expression d'un substrat sémantique appris à travers un procès d'apprentissage intersubjectif (Abrichtung)), et il produit, de cette manière, une sensibilité ultérieure. Wittgenstein se réfère au « voir comme » comme à ce qui dépasse le concept, en en conservant partiellement les traits :

35. L. Wittgenstein, Philosophische Untersuchungen, op. cit., II, xi, p. 178 ; tr. fr. : «Ce n'est que de quelqu'un qui est en mesure de faire aisément certaines applications de la figure du triangle qu'on dirait qu'il le voit tantôt comme ceci, tantôt comme cela. Le substrat de cette expérience vécue est la maîtrise d'une technique », op. cit. II, xi, p. 294.

36. Tr. fr. : «ceci comme sommet et cela comme base - puis ceci comme base, et cela comme sommet », op. cit. II, xi, p. 294.

37. L'intérêt de Wittgenstein pour les figures ambiguës et le changement de l'aspect d'une figure reflète son intérêt pour la psychologie de la forme (Gestalt Psychologie) et en particulier pour les théories de Wolfang Köhler. Cf. W. Köhler, Gestalt psychology, New York, Liveright, 1929, 1947². Le texte est paru aussi en 1933 dans la traduction allemande Psychologische Probleme.

38. L. Wittgenstein, Philosophische Untersuchungen, op. cit., II, xi, p. 181 ; traduction fr. : «Interpréter, c'est penser, c'est agir ; voir en revanche est un état », op. cit., II, xi, p. 299. 
«Es ist beinahe, als ob das 'Sehen des Zeichens in diesem Zusammenhang' ein Nachhall eines Gedankens wäre.

"Ein im Sehen nachhallender Gedanke" - möchte man sagen » 39.

Le "voir comme », donc, est comme un voir, et il n'est pas comme un voir, au sens qu'il n'appartient pas exclusivement au domaine de la perception en étant sa description, mais il se constitue, par contre, comme l'expression d'une pensée, (Gedankenausdruck), (cf. PU, II, XI, p. 168). Ainsi, remarquer un aspect, en saisir soudainement une propriété déterminée apparaît «halb Seherlebnis (expérience visuelle), halb ein Denken (pensée) », (PU, II, XI, p. 168) ${ }^{40}$. Il ne s'agit pas, alors, d'une problématique dont la solution serait à chercher au niveau des causes (de nature, par exemple, physiologique) : "Stellen wir uns das Sehen rätselhaft vor ! Ohne jederlei physiologische Erklärung » (BPP, I, § 963) $)^{41}$, mais d'un problème conceptuel pour lequel une explication de l'expérience vécue basée sur un critère médical-physiologique ne ferait que «das alte Problem verdecken, aber nicht lösen », (PU, II, XI, p. 181 $1^{42}$ ). En effet, pour ce concept modifié de sensation (modifizierter Empfindungsbegriff), on ne peut pas indiquer un organe sensoriel (Sinnesorgan) (cf. PU, II, XI, p. 179).

La différence entre une sensation ou une impression visuelle et ce type de sensibilité (« concept modifié de sensation») provient non seulement de sa relation privilégiée avec la pensée, mais aussi de la possibilité de saisir des relations entre objets. En d'autres termes, si l'impression visuelle renvoie aux propriétés d'un objet, le voir post-linguistique renvoie à des relations internes qui existent entre un objet et les autres objets :

«Der Farbe des Objekts entspricht die Farbe im Gesichtseindruck [...] der Form des Objekts die Form im Gesichtseindruck [...] - aber was ich im Aufleuchten des Aspekts wahrnehme, ist nicht eine Eigenschaft des Objekts, es ist eine interne Relation zwischen ihm und andern Objekten ${ }^{43}$.

Il s'agit donc d'un sentir qui se déplace dans la multiplicité des visages qui caractérisent le langage, en passant de l'un à l'autre et qui, dans la

39. Ibidem, tr. fr. : «C'est presque comme si "voir le signe dans ce contexte" était l'écho d'une pensée. "L'écho d'une pensée dans le voir" - pourrait on dire », op. cit., xi, p. 298.

40. Cf. aussi P. Bozzi, Vedere come. Commenti ai \$\$ 1-29 delle Osservazioni sulla filosofia della psicologia di Wittgenstein, Milan, Guerini e Associati, 1998. Ici Bozzi fait une analyse très précise de la notion de «voir comme » que nous trouvons dans les premiers vingt-neuf paragraphes du premier volume de Bemerkungen über die Philosophie der Psychologie.

41. Tr. fr.: «Représentons-nous le voir dans toute son énigme! Sans tout le bazar des explications physiologiques », op. cit., I, § 963.

42. Tr. fr. : « occulter l'ancien problème, non le résoudre », op. cit., II, xi, p. 299.

43. L. Wittgenstein, Philosophische Untersuchungen, op. cit., II, xi, p. 181 ; trad. fr. : « À la couleur de l'objet correspond la couleur de l'impression visuelle [...] - à la forme de l'objet, la forme de l'impression visuelle [...], mais ce que je perçois lors de l'apparition soudaine de l'aspect n'est pas une propriété de l'objet. C'est une relation interne entre lui et d'autres objets », op. cit., II, xi, p. 298. 
perspective wittgensteinienne, dépend d'un exercice de volonté (Wille) : «Der Aspekt ist dem Willen unterworfen. [...] Und daß der Aspekt (wenigstens bis $\mathrm{zu}$ einem gewissen Grade) willkürlich ist, scheint ihm wesentlich zu sein, wie auch der Vorstellung, daß sie es ist» (BPP, I, $\left.\S 899^{44}\right)$; «daß der Aspekt, wenigstens bis zu einem gewissen Grade, dem Willen untersteht » (BPP, I, §971 ${ }^{45}$ ); «Daß der Aspekt dem Willen untersteht, ist nicht eine, sein Wesen selbst nicht berührende, Tatsache » (BPP, I, § 976 $\left.{ }^{46}\right)$. Cependant, il ne faut pas céder à la tentation de surestimer ce facteur lié à la volonté en $^{4}$, soumis à un élément fondamental : l'apprentissage d'une technique d'application des concepts qui donnent la forme aux aspects (cf. PU, II, p. 178) ${ }^{48}$.

La nature du voir comme se rapprochant de la dimension conceptuelle, la possibilité de saisir des relations internes, c'est-à-dire, des ressemblances entre les objets, et la composante essentielle de volonté (quoique avec les limitations soulignées ci-dessus) représentent donc, selon Wittgenstein, les caractéristiques essentielles de l'expérience du « voir comme », et du « sentir comme », en général. En dehors de la vue, même les autres sens en réalité peuvent jouir d'une position spéciale à l'égard de la pensée. De nombreuses annotations wittgensteiniennes se référent, par exemple, à l'ouïe. Entendre une mélodie en connaissant le style du compositeur devient, ainsi, une manière totalement différente de l'entendre. Voir une image tantôt comme un lièvre (Hase) et tantôt comme un canard (Ente) ${ }^{49}$ (cf. PU, II, XI, p. 165-168), entendre la mélancolie d'une mélodie, reconnaître la joie (Freude) sur un visage (cf. BPP, I, § 927) ou comparer les mots selon des nuances subtiles de parfum (cf. PU, II, XI, p. 178) sont autant d'exemples d'une esthétique qui, en utilisant un lexique kantien, se constitue comme expérience sur la base d'une analytique.

44. Tr. fr. : «L'aspect est soumis à la volonté [...] Et que l'aspect soit (du moins jusqu'à un certain point) volontaire, cela paraît lui être essentiel, et essentiel aussi à l'imagination », op. cit., I, $\S 899$.

45. Tr. fr. : «le fait que l'aspect, du moins jusqu'à un certain point, est soumis à la volonté », op. cit., I, § 971 .

46. Tr. fr. : «Le fait que l'aspect soit soumis à la volonté n'est pas un simple fait, qui ne toucherait pas à son essence », op. cit., I, § 976.

47. Cf. A. Voltolini, Guida alla lettura delle Ricerche filosofiche di Wittgenstein, Bari, Laterza, 1998, p. 143.

48. Il ne faut pas oublier, en outre, qu'existent des limites à la variation de la vision des aspects imposée par les propriétés de l'objet même. Wittgenstein écrit: "Der Aspekt untersteht dem Willem". Er ist nicht Erfahrungssatz). Es hat Sinn, zu sagen "Sieh diesen Kreis als Loch, nicht als Scheibe"; aber nicht "Sieh ihn als Viereck", oder "Sieh ihn rot" » (BPP, II, § 545) ; tr. fr. : " "L'aspect est soumis à la volonté." Ce n'est pas une proposition d'expérience. Il y a du sens à dire: "Vois ce cercle comme un trou, non comme un disque" ; mais il n'y en a pas à dire : "Vois-le comme un quadrilatère" ou "Vois-le rouge" ", op. cit., II, $\S 545$.

49. Wittgenstein emprunte à Joseph Jastrow la figure qui peut être vue tantôt comme une tête de lièvre, tantôt comme une tête de canard (cf. J. Jastrow, J., Fact and Fable in Psychology, Londres, Macmillan and Co., 1901, p. 295). 
On a défini ainsi comme post-linguistique la perception (visuelle, auditive, tactile etc.) qui a été modifiée par une présupposition conceptuelle, et qui serait donc inconcevable sans une familiarité préliminaire avec un réseau sémantique de significations. Dans cette perspective Wittgenstein parle de la signification secondaire (post-linguistique) comme d'une possibilité qui jaillit à la suite de la maîtrise d'une technique et des usages primaires du langage. Cependant, elle n'est pas synonyme de signification métaphorique (übertragen) :

«Wenn ich sage: 'Der Vokal e ist für mich gelb', so meine ich nicht 'gelb' in übertragener Bedeutung - denn ich könnte, was ich sagen will, gar nicht anders als mittels des Begriffs, 'gelb' ausdrücken $»^{50}$.

Le même phénomène a lieu aussi quand nous classifions les jours de la semaine en fett ou mager (cf. PU, II, XI, p. 184), et dans tous les autres exemples qu'il est possible de créer à partir de notre familiarité avec une grammaire déterminée. Sensibilité post-linguistique et signification secondaire se réfèrent toutefois à une perception immédiate dans laquelle il n'y a pas de déphasage diachronique avec l'impression sensorielle primaire, ni de variation d'intensité. Avec les traits d'un visage dessinés d'une certaine manière, par exemple, j'aperçois aussi, en même temps, avec égale spontanéité, la ressemblance du visage avec un autre ou j'en reconnais les expressions de joie ou de douleur. Dans l'acte même du voir, et non pas pendant l'instant suivant et par une réflexion, je perçois sensiblement la façon de se montrer de la forme, c'est-à-dire «der Ausdruck des Aspektwechsels ist der Ausdruck einer neuen Wahrnehmung, zugleich mit dem Ausdruck der unveränderten Wahrnehmung »(PU, II, XI, p. 167 $7^{51}$ ). «Voir comme »n'est ainsi pas une perception objectale d'une interprétation conceptuelle qui reste indifférente au changement d'aspect. Il est plutôt un voir (sehen) en accord avec une interprétation (Deutung); nous voyons une image en même temps que nous l'interprétons : «Aber wir können auch die Illustration einmal als das eine, einmal als das andere Ding sehen.- Wir deuten sie also, und sehen sie, wie wir sie deuten » (PU, II, XI, p. 165) ${ }^{52}$.

50. L. Wittgenstein, Philosophische Untersuchungen, op. cit., II, xi, p. 184 ; tr. fr. : «Quand je dis : "Pour moi, la voyelle $e$ est jaune", je ne comprends pas "jaune" dans une signification figurée - car il me serait impossible d'exprimer ce que je souhaite dire autrement que par le concept "jaune" », op. cit., II, xi, p. 304.

51. Tr. fr. : «L'expression du changement d'aspect est l'expression d'une nouvelle perception, et en même temps celle d'une perception inchangée », op. cit., II, xi, p. 278.

52. Tr. fr. : « Mais il nous est également possible de voir l'illustration une fois comme telle chose, une autre fois comme telle autre chose. - Nous l'interprétons donc, et nous la voyons comme nous l'interprétons », op. cit., II, p. 275. À propos de cette opposition entre interpréter et voir en accord avec une interprétation, il est possible de mettre en évidence un parallélisme avec ce que Wittgenstein soutient sur le thème de « suivre une règle », c'est-à-dire que : « daß es eine Auffassung einer Regel gibt, die nicht eine Deutung ist» (PU I, § 201). Comme souligné par Baker et Hacker dans Scepticism, Rules and Language, puisque les arguments de la non-interpretativité, du «voir comme » et du «suivre une règle » sont discutés ensemble 
Il est ainsi évident que dans l'espace du post-linguistique, de la même façon qu'à l'autre extrême du jeu, dans l'espace du pré-linguistique, il n'y a pas de place pour l'interprétation diachronique, le raisonnement conceptuel ou les procédés analogiques. Wittgenstein dit ici : «Keinen Grund, das zu glauben » (PU, II, XI, p. $183^{53}$ ). L'activité rationnelle de l'interprétation est ainsi remplacée par l'état (Zustand) de ce sentir particulier qui ne se réfère pas aux organes de sens (Sinnesorgan), mais aux concepts (Begriffe) (cf. PU, II, XI, p. 178). Autrement dit, dans l'expérience du « sentir comme » il n'y a pas d'interprétations différentes qui se superposent aux données de la perception, mais des formes différentes d'organisation conceptuelle des données. Si le phénomène physiologique (par exemple l'image rétinienne) reste inchangé, au contraire, l'élément structural de la pensée qui donne forme, en l'organisant, à la perception, change. Même dans l'espace postlinguistique, donc, il n'est pas permis de se déplacer selon les directions de la rationalité, du calcul ou dans les termes du vrai et du faux : «Das Wissen, die Meinung, haben keinen Gesichtsausdruck» (BPP, I, § 928 ${ }^{54}$ ). La gaieté perçue sur un visage ou la reconnaissance d'une souffrance n'appartiennent pas à la sphère de la réflexion conceptuelle, mais à celle du contact direct, de la knowledge by acquaintance ou, pour utiliser un lexique aristotélicien, du thigein $^{55}$.

\section{Corps et reconnaissance}

Selon mon interprétation, à l'intérieur de la perspective wittgensteinienne de la «reconnaissance », la notion de «corps», en tant que lieu d'une naturalité instinctive et non médiatisée par le concept, assume un rôle essentiel. La reconnaissance d'Autrui, de sa souffrance ou de sa joie, de sa stupeur ou de sa tranquillité à travers l'observation des traits du visage, par exemple, est ce qui permet la reconnaissance de l'esprit ou de l'âme de l'autre («Meine Einstellung zu ihm ist eine Einstellung zur Seele», PU, II, IV, p. $152^{56}$ ) et, donc, la reconnaissance de l'autre comme être

par Wittgenstein dans un manuscrit préparatoire aux Untersuchungen, il est correct de supposer qu'entre les deux thèmes il y ait bien plus qu'une analogie (cf. G. Baker, P. Hacker, Scepticism, Rules and Language, Oxford, Blackwell, 1984, p. 14-15).

53 Tr. fr. : «aucune raison de le croire », op. cit., II, xi, p. 302.

54. Tr. fr. : «Le savoir, l'intention, n'ont pas d'expression faciale », op. cit., I, § 928.

55. Cf. Aristote, Métaphysique, in Aristotelis opera omnia, Firmin-Didot, Paris, 1848. Ici, à propos des objets simples, Aristote s'interroge sur leur être et leur non-être, sur leur vérité ou fausseté. Et si pour les entités composées la prédication correcte correspond au vrai et celle incorrecte au faux, pour les objets simples (tà asyntheta) c'est différent : pour ces derniers, le vrai consiste à avoir un contact direct [thigein] avec une chose et à l'énoncer [phanai], par contre ne pas avoir de contact direct avec celle-ci signifie de ne pas la connaître.

56. Tr. fr. : « Mon attitude à son égard est une attitude à l'égard d'une âme », op. cit., II, ibid., p. 253. 
humain. Le corps de l'autre, en tant que lieu des gestes, des regards, des expressions les plus subtiles se transforme en un miroir qui permet de voir l'autre, de l'entendre, et ainsi de le reconnaître, à travers une évidence définie par Wittgenstein comme «impondérable » (unwägbar) : des Tons.

«Zur unwägbare Evidenz gehören die Feinheiten des Blicks, der Gebärde,

Ich mag den echten Blick der Liebe erkennen, ihn vom verstellten unterscheiden $»^{57}$.

Le corps, et le visage en particulier, représente un élément essentiel du jeu intersubjectif, fondamental à la reconnaissance consciente (et non à la connaissance épistémique: «Der Unterschied zwischen 'knowing' und 'being aware of ${ }^{, 58}$ », BPP, II, § 45 et Z, § 85) de l'autre :

«Der menschliche Körper ist das beste Bild der menschlichen Seele ${ }^{59}$.

Le corps est donc la meilleure image de l'âme humaine, et cela non parce qu'il la représente mais plutôt parce qu'il l'exprime. Le miroir du corps ne produit pas des représentations secondaires, c'est-à-dire des images virtuelles de l'âme (ou de l'esprit), mais il permet d'y accéder sans médiations : «Man sieht Gemütsbewegung » (BPP, II, §57060). Dans la perspective wittgensteinienne le trait distinctif des êtres humains n'est pas un élément d'intelligence cognitive, mais plutôt une intelligence incarnée dont la corporéité se fait le véhicule. Cela ne fait pas du corps seulement un «porteur » d'états mentaux, mais aussi l'expression de notre présence à l'intérieur de formes de vie (de notre façon d'être au monde), où notre substrat culturel et acquis au cours du temps se croise avec nos compétences perceptives et motrices.

De tout ce que l'on a dit, il résulte que c'est seulement au niveau de la science médicale et biologique, anatomique et physiologique, que le corps peut être additionné aux autres corps (la relation entre corps est alors réduite à une relation entre choses), mais ceci n'est pas possible pour le corps-âme

57. L. Wittgenstein, Philosophische Untersuchungen, op. cit., II, ix, p. 194 ; trad. fr. : « La subtilité du regard, des gestes et du ton relève de l'évidence impondérable », op. cit., II, xi, p. 319. Cf. aussi : «Die Frage liegt nahe : Könnten wir uns Menschen denken, die nie etwas als etwas sähen? Würde diesen ein wichtiger Sinn fehlen; ähnlich als wären sie farbenblind oder als fehlte ihnen absolutes Gehör ? Nennen wir solche Menschen einmal "gestaltblind" oder "aspektblind" » (BPP, II, § 478) ; tr. fr. «La question suivante s'offre d'elle-même : Ne pourrions-nous imaginer des hommes qui ne verraient jamais quelque chose comme quelque chose ? Leur manquerait-il un sens important, comme s'ils étaient daltoniens ou que l'oreille absolue leur fît défaut? Nommons de tels hommes "aveugles à l'aspect" », op. cit., II, § 478.

58. Tr. fr. : «La différence entre "knowing" et "being aware" ». En anglais dans le texte de Wittgenstein.

59. L. Wittgenstein, Philosophische Untersuchungen, op. cit., II, ibid., p. 152 ; tr. fr. : « Le corps humain est la meilleure image de l'âme humaine », op. cit., II, ibid., p. 254.

60. Tr. fr. : « On voit l'émotion », op. cit., II, § 570. 
au sens wittgensteinien. Dans cette perspective, le corps se transforme non pas en ce qui nous sépare (comme le sceptique le soutient), mais plutôt en ce qui nous unit, en constituant le fondement concret de l'intersubjectivité sociale. La relation Moi-Autrui est déplacée sur la dimension corporelle de la spontanéité qui n'a pas besoin de se fonder sur des justifications de type cognitif. Dans les relations intersubjectives, selon Wittgenstein, la conjecture et le raisonnement conceptuel laissent la place à la réaction immédiate, dans le cas de l'instinct naturel autant que dans celui de la sensibilité postlinguistique provenant d'une expérience sémantique déterminée.

La notion de «corps » en tant que «lieu de la spontanéité prélinguistique et post-linguistique » permet alors de confirmer d'une façon encore plus évidente le déplacement de perspective que Wittgenstein opère en rapport au sujet de l'intersubjectivité d'un niveau épistémique à un niveau anthropologique, c'est-à-dire du niveau de la connaissance à celui de la reconnaissance immédiate d'Autrui.

\section{Références bibliographiques}

\section{Oeuvres de Wittgenstein :}

[BPP] - Bemerkungen über die Philosophie der Psychologie. Remarks on the Philosophy of Psychology [1946-1949; 1947-1948], édité par G. E. M. Anscombe et G. H. Von Wright, trad. par G. E. Anscombe, 2 vol., Oxford, Basil Blackwell, 1980, 1988² ; tr. fr. Remarques sur la Philosophie de la Psychologie, par G. Granel, T.E.R., Mauvezin, 1994.

[LS] - Lezte Schriften über die Philosophie der Psychologie. Last writings on the Philosophy of Psychology. vol. 1 Vorstudien zum zweiten Teil der "Philosophische Untersuchungen". Preliminary Studies for Part II of the "Philosophical Investigation" [1948-1949] ; vol. 2 Das Innere und das Äussere. The Inner and Outer [1948-1951], ed. par G. H. von Wright et Hikky Nyma, trad. par C. J. Luckhardt et M. A. E. Aue, Chicago, The University of Chicago Press, 1982, 1990² ; tr. fr. vol. I Études préparatoires à la seconde partie des "Recherches philosophiques" par G. Granel, T.E.R., Mauvezin, 1985 ; tr. fr. vol. 2 L'intérieur et l'extérieur. Derniers écrits sur la philosophie de la psychologie, par G. Granel, T.E.R., Mauvezin, 2000.

[PG]-Philosophische Grammatik. Philosofical Grammar [1929-1934; 1934-1935], in Werkausgabe Band 4, Frankfurt am Main, Suhrkamp, 1984 ; tr. fr. Grammaire philosophique, par M.-A. Lescourret, Gallimard, Paris, 1980. 
[PU]-Philosophische Untersuchungen. Philosophical Investigations, trad. angl. par G. E. Anscombe, Oxford, Blackwell, 1953, 2001 $1^{3}$; tr. fr. Recherches philosophiques, par F. Dastur, M. Élie, J.-L. Gautero, D. Janicaud, E. Rigal, Gallimard, Paris, 2004.

[TLP]-Tractatus Logico-Philosophicus [1918-1921], in Werkausgabe Band 1, sous la direction de G. H. Von Wright, et G. E. M. Anscombe, Frankfurt am Main, Suhrkamp, 1964, 1989² ; tr. fr. Tractatus logicophilosophicus, par G.-G. Granger, Gallimard, Paris, 1993.

[UG]-Über Gewissheit. On Certainty [1950-1951], édité par G. E. M. Anscombe et G. H. Von Wright, tr. par. G. E. Anscombe et Denis Paul, New York, Harper \& Row, 1972 ; tr. fr. De la certitude, par J. Fauve, Gallimard, Paris 1965, $1976^{2}$.

[Z] - Zettel [1929-1948, 1945-1948], édité par G. E. M. Anscombe et G. H. Von Wright, trad. par G. E. M. Anscombe, Berkeley, University of California Press, 1970, $1975^{2}$; tr. fr. Fiches, par J. Fauve, Gallimard, Paris 1970.

\section{Oeuvres générales :}

Aristote, Métaphysique, in Aristotelis opera omnia, Paris, Firmin-Didot, 1848.

Baker G., Hacker P., Scepticism, Rules and Language, Oxford, Blackwell, 1984.

Benoist J., Les limites de l'intentionnalité. Recherches phénoménologiques et analytiques, Paris, Vrin, 2005.

Borutti S., «Percezione e immagine. Un'estetica per le scienze umane », in "Aut aut", 313-314, 2003, p. 185-198.

Bouveresse J., Le mythe de l'intériorité. Expérience, signification et langage privé chez Wittgenstein, Paris, Minuit, 1976, $1987^{2}$.

Bozzi P., Vedere come. Commenti ai \$\$1-29 delle Osservazioni sulla filosofia della psicologia di Wittgenstein, Milan, Guerini e Associati, 1998.

Cavell S., The Claim of Reason. Wittgenstein, Skepticism, Morality, and Tragedy, New York, Oxford University Press, 1979.

Chauviré Ch., Voir le visible. La seconde philosophie de Wittgenstein, Paris, PUF, 2003.

Descartes R., Discours de la méthode (1647), Paris, Hatier, 1999.

Dretske F., Seeing and Knowing, Chicago, The University of Chicago Press, 1969.

Frege G., Funktion und Begriff (1891), H. Pohle, Iéna, 1891, tr. fr. Fonction et concept par C. Imbert, in Écrits logiques et philosophiques, Paris, Seuil, 1971, p. 80-101.

Goethe J. W., Faust I, 1808. 
Jastrow J., Fact and Fable in Psychology, Londres, Macmillan and Co., 1901.

Köhler W., Gestalt psychology, New York, Liveright, 1929, $1947^{2}$.

Malcolm N., Problems of Mind. Descartes to Wittgenstein, New York, Harper \& Row, 1971.

Malcolm N., Wittgenstein: the Relation of Language to Instinctive Behaviour, Swansea, University College of Swansea, 1981.

Mazzeo M., Virno P., «Il fisiologico come simbolo del logico. Wittgenstein fisionomo », in Sensibilità e linguaggio. Un seminario su Wittgenstein, Macerata, Quodlibet, 2002, 119-155.

Perissinotto L., «Wittgenstein e il problema degli altri », in "Aut aut”, 51, 2001, p. 16-25.

Sparti D., L'importanza di essere umani. Etica del riconoscimento, Milan, Feltrinelli, 2003.

Voltolini A., Guida alla lettura delle Ricerche filosofiche di Wittgenstein, Bari, Laterza, 1998. 